\title{
Robust stabilisation of multivariable feedback systems with desired performance requirement
}

M.-H. Tu

C. - M. Lin

Indexing terms: Control systems, Internal stability, Robust stabilisation

\begin{abstract}
A design criterion is developed to achieve the following goals simultaneously: (i) input-output decoupling of multivariable feedback systems; (ii) complete and arbitrary closed-loop pole assignment; (iii) desired zero assignment for reference signal tracking; and (iv) robust stabilisation of multivariable feedback systems subjected to time-varying nonlinear uncertainties. Thus, the requirements of performance as well as stability robustness of a multivariable feedback system will be simultaneously met by employing this design criterion. Moreover, by minimising $H^{\infty}$-norm of each channel of the closed-loop transfer matrix, we can obtain the robustness optimisation of the system, i.e., we can predict the maximum slope of the sector-bounded nonlinear uncertainties that can be tolerated in each channel of the system. A practical example, the lateral flight control of $\mathrm{CCV}$ (control configured vehicle), is given to illustrate the validity of the proposed design algorithm.
\end{abstract}

\section{Introduction}

In control systems the pole dominates the transient response as well as the system stability and so many studies $[1,2]$ have addressed pole assignment design. In addition, since the zero of a system plays an important role in the interaction between the system and its external environment, a great deal of research $[3,4]$ has gone into achieving reference signal tracking by assigning appropriate zeros. Thus, the pole-zero assignment of a control system is important for a system to achieve desired performance requirement. Another important control strategy is the robust stabilisation problem, i.e., the ability to maintain system stability under plant uncertainties. Cruz et al. [5] have discussed the robust stabilisation of linear feedback systems with time-varying nonlinear perturbations in terms of the roles of singular values. However their results are valid only when the plant and the controller are stable. Other work $[6,7]$ uses the spectral norm to formulate an upper bound on the largest singular value of the closed-loop transfer matrix to guarantee

Paper 8696D (C8), first received 26th January 1991 and in revised form 2nd January 1992

M.-H. Tu is with the Institute of Electronics, National Chiao-Tung University, 1001 Ta-Hsueh Road, Hsin-Chu, 30050 Taiwan, Republic of China

C.-M. Lin is with the Chung-Shan Institute of Science and Technology, PO Box 90008-6-5, Lung-Tan, Tao-Yuan 32500, Taiwan, Republic of China

IEE PROCEEDINGS-D, Vol. 139, No. 3, MAY 1992 robust stability of a multivariable control sytem under parameter variation. Dickman and Sivan [8] have shown that among the different, not necessarily diagonal, closedloop transfer matrices which have the same diagonal elements, the diagonal closed-loop transfer matrix has the greatest robustness for a multivariable system. Allowable perturbations are discussed in References 9 and 10 for maintaining stability of uncertain systems. These results are concerned only with stability robustness, they do not deal with the robustness for maintaining a certain performance. Robustness results which do address the performance problem are found in References 11 and 12.

The primary purpose of this paper is to outline a design criterion for achieving the input-output decoupling (i.e. obtaining the diagonal closed-loop transfer matrices) of multivariable feedback systems and the robust stabilisation of systems subjected to time-varying nonlinear uncertainties with desired pole-zero assignment. By appropriately assigning the poles and zeros of the sensitivity matrix and simultaneously satisfying the robustness requirement, the proposed design algorithm ensures:

(i) input-output decoupling of multivariable feedback systems;

(ii) complete and arbitrary closed-loop pole assignment;

(iii) desired zero assignment for reference signal tracking;

(iv) robust stabilisation of multivariable feedback systems subjected to time-varying nonlinear uncertainties.

Moreover, by minimising $H^{\infty}$-norm of each channel of the closed-loop transfer matrix, we can obtain the robustness optimisation of the system, i.e., we can predict the maximum slope of the sector-bounded nonlinear uncertainties that can be tolerated in each channel of the system.

The notations used in this paper are as follows: $\operatorname{deg}(*)$ denotes the degree of the polynomial $*$. diag $(\cdot)$ denotes a diagonal matrix. $\|\cdot\|$ denotes the Euclidean norm. The $H^{\infty}$-norm of a transfer function $G(s)$ is

$$
\|G\|_{\infty}=\sup |G(j \omega)|, \quad \forall \omega \in[0, \infty]
$$

and BIBO stands for bounded-input bounded-output.

\section{Problem formulation}

Consider the multivariable feedback system subjected to time-varying nonlinear uncertainties shown in Fig. 1, where $\bar{P}, P$ and $C \in C^{n \times n}$ denote the practical plant, nominal plant and the controller, respectively. The timevarying nonlinear uncertainty $\Delta N[u(t), t]=$ diag 
$\left[\Delta N_{1}[u(t), t], \ldots, \Delta N_{n}[u(t), t]\right]$ is a real-valued matrix of the control signal $u(t) \in R^{n}$ and $t \in[0, \infty]$ satisfying the following:

(a) $\Delta N_{i}[0, t]=0, i=1, \ldots, n$, for $t \in[0, \infty]$

(b) $\Delta N_{i}[u(t), t], i=1, \ldots, n$ are measurable functions of $t$ for all measerable $u(t) ;$ and

(c) there exist finite constants $\rho_{i}>0, i=1, \ldots, n$ with the property

$\left|\Delta N_{i}[u(t), t]\right| \leqslant \rho_{i}\|u(t)\|, i=1, \ldots, n \forall\|u(t)\|<\infty \quad(1)$

and the nonlinear uncertainties within the sectors having slopes $\rho_{i}, i=1, \ldots, n$ are denoted as $\Delta N_{i}[u(t), t]$,

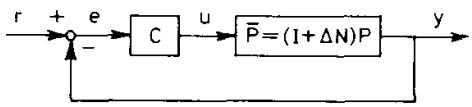

Fig. 1 Multivariable feedback system with nonlinear uncertainties

$i=1, \ldots, n$, and $\rho_{i}\|u(t)\|, i=1, \ldots, n$ are the sector bounds of the nonlinear uncertainties.

Let the nominal plant of the system in Fig. 1 be factorised as

$$
P(s)=A^{-1}(s) B(s)=B_{1}(s) A_{1}^{-1}(s)
$$

where the pairs $(A(s), B(s))$ and $\left(B_{1}(s), A_{1}(s)\right)$ are the left and right coprime polynomial matrix factorisations of the plant, respectively. The sensitivity matrix is defined as

$$
S(s)=(I+P(s) C(s))^{-1}
$$

then the tracking error signal $E(s)$ can be obtained as

$$
E(s)=S(s) R(s)
$$

where $R(s)$ is the reference signal. Let the reference signal $R(s)=\left[r_{1}(s), r_{2}(s), \ldots, r_{n}(s)\right]^{T}$ and let the zeros of the polynomials $m_{i}(s)$ be the poles of $r_{i}(s)$ in $\operatorname{Re}[s] \geqslant 0$ for $i=1, \ldots, n$.

For the input-output decoupling, reference signal tracking and desired closed-loop pole assignment, the sensitivity matrix must be of the form

$$
\begin{aligned}
S(s) & =\operatorname{diag}\left[s_{1}(s), \ldots, s_{n}(s)\right] \\
& =\operatorname{diag}\left[\frac{w_{1}(s) m_{1}(s)}{g_{1}(s)}, \ldots, \frac{w_{n}(s) m_{n}(s)}{g_{n}(s)}\right]
\end{aligned}
$$

where $g_{i}(s), i=1, \ldots, n$ are Hurwitz polynomials with desired closed-loop poles and $w_{i}(s), i=1, \ldots, n$ are undetermined polynomials which should be determined to satisfy the internal stability constraints.

\section{Pole-zero assignment control law design}

For application to any stable or unstable, minimum or nonminimum phase system, we first derive a pole-zero assignment control law which satisfies the internal stability of the multivariable feedback system in the nominal case, i.e. $\Delta N[u(t), t]=0$, for all $t \in[0, \infty]$.

Lemma 1 [13]: Suppose $\operatorname{det}(A(s))$ and $\operatorname{det}\left(B_{1}(s)\right)$ have no common zero in $\operatorname{Re}[s] \geqslant 0$. Then $S(s)$ is internally stable if and only if $S(s)$ is analytic in $\operatorname{Re}[s] \geqslant 0$ and for some appropriately dimensioned stable rational matrices $X(s)$ and $Y(s)$ such that

$$
\begin{aligned}
& S(s)=Y(s) A(s) \\
& I-S(s)=B_{1}(s) X(s)
\end{aligned}
$$

From eqns. 6 and 7 , we have

$$
\begin{aligned}
& S(s) A^{-1}(s)=Y(s) \\
& B_{1}^{-1}(s)(I-S(s))=X(s)
\end{aligned}
$$

Equivalently, $S(s)$ is internally stable if and only if $S(s)$, $S(s) A^{-1}(s)$ and $B_{1}^{-1}(s)(I-S(s))$ are all analytic in $\operatorname{Re}[s] \geqslant 0$.

Remark: If $S(s)$ satisfies the requirements of internal stability, then we can directly obtain the controller as

$$
C(s)=P^{-1}(s) S^{-1}(s)(I-S(s))
$$

without worrying about any unstable hidden mode.

Assume

$$
\begin{aligned}
A^{1}(s) & =\left[\begin{array}{ccc}
a_{11}(s) & \cdots & a_{1 n}(s) \\
\vdots & & \vdots \\
a_{n 1}(s) & \cdots & a_{n n}(s)
\end{array}\right] \\
B_{1}^{-1}(s) & =\left[\begin{array}{ccc}
b_{11}(s) & \cdots & b_{1 n}(s) \\
\vdots & & \vdots \\
b_{n 1}(s) & \cdots & b_{n n}(s)
\end{array}\right]
\end{aligned}
$$

From eqns. 5, 11 and 12 , we obtain

$$
\begin{gathered}
S(s) A^{-1}(s)=\left[\begin{array}{ccc}
s_{1}(s) a_{11}(s) & \cdots & \left.s_{1}(s) a_{1 n} s\right) \\
\vdots & & \vdots \\
s_{n}(s) a_{n 1}(s) & \cdots & s_{n}(s) a_{n n}(s)
\end{array}\right] \\
B_{1}^{-1}(s)(I-S(s))=\left[\begin{array}{ccc}
\left(1-s_{1}(s)\right) b_{11}(s) & \cdots & \left(1-s_{n}(s)\right) b_{1 n}(s) \\
\vdots & & \vdots \\
\left(1-s_{1}(s)\right) b_{n 1}(s) & \cdots & \left(1-s_{n}(s)\right) b_{n n}(s)
\end{array}\right]
\end{gathered}
$$

Define the polynomials

$$
\alpha_{i}(s)=\prod_{l=1}^{\lambda_{i}}\left(s-p_{i l}\right)^{\sigma_{i l}}, \quad i=1, \ldots, n
$$

where $\lambda_{i}$ is the number of distinct poles $p_{i l}$ of the ith row of $A^{-1}(s)$ in $\operatorname{Re}[s] \geqslant 0$ and $\sigma_{i l}$ is the greatest multiplicity of each pole $p_{i l}$ which appears in any element of the $i$ th row of $A^{-1}(s)$. Similarly, we define the polynomials

$$
\beta_{i}(s)=\prod_{l=1}^{\delta_{i}}\left(s-q_{i i}\right)^{n_{i i}}, \quad i=1, \ldots, n
$$

where $\delta_{i}$ is the number of distinct poles $q_{i l}$ of the $i$ th column of $B_{1}^{-1}(s)$ in $\operatorname{Re}[s] \geqslant 0$ and $\eta_{i l}$ is the greatest multiplicity of each pole $q_{i l}$ which appears in any element of the $i$ th column of $B_{1}^{-1}(s)$. Then we obtain the following lemma:

Lemma 2: For the nominal system (i.e. $\Delta N[u(t), t]=0$ ) shown in Fig. 1, if $\alpha_{i}(s)$ and $\beta_{i}(s)$ are coprime for $i=1, \ldots$, $n$, then $S(s)=\operatorname{diag}\left[s_{1}(s), \ldots, s_{n}(s)\right]$ is internally stable if and only if the following conditions hold:

(i) $s_{i}(s)$ is analytic in $\operatorname{Re}[s] \geqslant 0$ for $i=1, \ldots, n$;

(ii) the numerator polynomial of $s_{i}(s)$ is divisible by $\alpha_{i}(s)$ for $i=1, \ldots, n$;

(iii) the numerator polynomial of $1-s_{i}(s)$ is divisible by $\beta_{i}(s)$ for $i=1, \ldots, n$.

Remark: If there exists any pair $\left(\alpha_{i}(s), \beta_{i}(s)\right)$ which is not coprime, then it is impossible for $S(s)$ to achieve the internal stability.

From condition (ii) of Lemma 2, the numerator polynomial of $s_{i}(s)$ must contain $\alpha_{i}(s)$, and from eqn. 5 , the

IEE PROCEEDINGS-D, Vol. 139, No. 3, MAY 1992 
numerator of $s_{i}(s)$ must also contain $m_{i}(s)$. Thus the numerator of $s_{i}(s)$ must contain the least common multiplier of $x_{i}(s)$ and $m_{i}(s)$ for $i=1, \ldots, n$, i.e.,

$$
\begin{aligned}
S(s) & =\operatorname{diag}\left[\frac{w_{1}(s) m_{1}(s)}{g_{1}(s)}, \ldots, \frac{w_{n}(s) m_{n}(s)}{g_{n}(s)}\right] \\
& =\operatorname{diag}\left[\frac{l_{1}(s) z_{1}(s)}{g_{1}(s)}, \ldots, \frac{l_{n}(s) z_{n}(s)}{g_{n}(s)}\right]
\end{aligned}
$$

where $z_{i}(s), i=1, \ldots, n$ are the least common multipliers of $x_{i}(s)$ and $m_{i}(s)$ while $l_{i}(s), i=1, \ldots, n$ are undetermined polynomials. To satisfy the requirement of causality of the closed-loop system, the sensitivity matrix must be proper, i.e.,

$$
\operatorname{deg}\left(g_{i}(s)\right) \geqslant \operatorname{deg}\left(l_{i}(s)\right)+\operatorname{deg}\left(z_{i}(s)\right) \quad i=1, \ldots, n
$$

From eqn. 17, we have

$$
1-s_{i}(s)=\frac{g_{i}(s)-l_{i}(s) z_{i}(s)}{g_{i}(s)} \quad i=1, \ldots, n
$$

and from condition (iii) of Lemma 2, the numerator of $1-s_{\mathbf{i}}(s)$ must contain $\beta_{i}(s)$. Thus we have

$$
h_{i}(s) \equiv g_{i}(s)-l_{i}(s) z_{i}(s)=\beta_{i}(s) f_{i}(s) \quad i=1, \ldots, n
$$

where $f_{i}(s), i=1, \ldots, n$ are undetermined polynomials.

Theorem 1: The solution of $l_{i}(s)$ in eqn. 20 exists if and only if $m_{i}(s)$ is coprime with $\beta_{i}(s)$, for $i=1, \ldots, n$, respectively.

Proof: (If): Since $m_{i}(s)$ is coprime with $\beta_{i}(s)$, and $\alpha_{i}(s)$ is coprime with $\beta_{i}(s)$, so that $z_{i}(s)$ is coprime with $\beta_{i}(s)$; and since $g_{t}(s)$ is a Hurwitz polynomial and is coprime with $\beta_{i}(s)$, so that the solution of $l_{i}(s)$ in eqn. 20 exists, for $i=1, \ldots, n$, respectively.

(Only if): By contradiction, suppose $m_{i}(s)$ is not coprime with $\beta_{i}(s)$, so that $z_{i}(s)$ and $\beta_{i}(s)$ must have a common factor with zeros in $\operatorname{Re}[s] \geqslant 0$. And since $g_{i}(s)$ is a Hurwitz polynomial and is coprime with $\beta_{i}(s)$, hence the solution of $l_{i}(s)$ in eqn. 20 does not exist, for $i=1, \ldots$, $n$, respectively. This contradicts to the fact that the solution of $l_{i}(s)$ in eqn. 20 exists, so that $m_{i}(s)$ must be coprime with $\beta_{i}(s)$. $Q E D$.

Remark: If $l_{i}(s)$ exists and the number of undetermined parameters of $l_{i}(s)$ is equal to $\operatorname{deg}\left(\beta_{i}(s)\right)$, then the solution of $l_{i}(s)$ in eqn. 20 is unique for $i=1, \ldots, n$, respectively.

By solving eqn. 20 , we obtain $l_{i}(s)$, i.e. $S(s)$ is obtained. Then the controller can be derived as

$$
C(s)=P^{-1}(s) S^{-1}(s)(I-S(s))
$$

Moreover, if $l_{i}(s)$ exists and the number of undetermined parameters of $l_{i}(s)$ is greater than $\operatorname{deg}\left(\beta_{i}(s)\right)$, then the solutions of $l_{i}(s)$ are not unique for $i=1, \ldots, n$, respectively. This leads to an over-parameterised solution and the free parameters of $l_{i}(s)$ can be determined according

\section{Example}

A practical example, the lateral flight control of $\mathrm{CCV}$ (control configured vehicle) [14], is given to illustrate the validity of the proposed design algorithm.

The nominal lateral dynamics of $\mathrm{T} 2 \mathrm{CCV}$ can be decribed as follows:

$$
\left[\begin{array}{c}
\dot{\beta} \\
\dot{\phi} \\
\dot{p} \\
\dot{r} \\
\psi
\end{array}\right]=\left[\begin{array}{cllll}
-0.259 & 0.039 & 0 & -1 & 0 \\
0 & 0 & 1 & 0 & 0 \\
-65.05 & 0 & -3 & 2.04 & 0 \\
-7.88 & 0 & -0.05 & -0.47 & 0 \\
0 & 0 & 0 & 1 & 0
\end{array}\right]\left[\begin{array}{l}
\beta \\
\phi \\
p \\
r \\
\psi
\end{array}\right]+\left[\begin{array}{ccl}
-0.09 & 0.121 & 0.081 \\
0 & 0 & 0 \\
151 & 46.2 & 7.106 \\
3.654 & -15.94 & 6.206 \\
0 & 0 & 0
\end{array}\right]\left[\begin{array}{c}
\delta_{f} \\
\delta_{r} \\
\delta_{v_{c}}
\end{array}\right]
$$

IEE PROCEEDINGS-D, Vol. I39, No. 3, MAY 1992 to some specific performance criteria. In the following, $l_{i}(s), i=1, \ldots, n$ are determined to satisfy the stability robustness requirement, and furthermore to minimise the $H^{\infty}$-norm of each channel of the closed-loop transfer function to obtain the robustness optimisation of the system.

\section{Robust stabilisation of time-varying nonlinear} uncertainties

In the following, we consider the robust stabilisation of the feedback system subjected to sector-bounded nonlinear uncertainties.

Theorem 2 [6]: The feedback system in Fig. 1 is BIBO stable if

(i) $S(s)$ is internally stable and

(ii) $\rho_{i}\left\|1-s_{i}\right\|_{x_{0}}<1$ for $i=1, \ldots, n$ or

$$
\sup _{w \in[0, \infty]} \rho_{i}\left|1-s_{i}(j \omega)\right|<1 \quad i=1, \ldots, n
$$

Proof: For the proof of Theorem 2, refer to Reference 6.

From the above analysis, the objective of the robust stabilisation control design of feedback systems subjected to sector-bounded nonlinear uncertainties is to adjust the controller $C(s)$ such that the sensitivity function $S(s)$ satisfies the internal stability of Lemma 2 with desired pole-zero assignment and the robust stability given in eqn. 22.

Thus, we obtain the following design algorithm for the robust stabilisation of multivariable feedback systems with desired performance requirement:

Step 1: Perform the factorisation $P(s)=A^{-1}(s) B(s)=$ $B_{1}(s) A_{1}^{-1}(s)$ and calculate $A^{-1}(s)$ and $B_{1}^{-1}(s)$, then determine $\alpha_{i}(s)$ and $\beta_{i}(s)$, for $i=1, \ldots, n$. And determine $m_{i}(s)$ and $z_{i}(s)$ as in eqn. 17.

Step 2: Choose diagonal sensitivity matrix $S(s)=$ diag $\left[s_{1}(s), \ldots, s_{n}(s)\right]$.

Step 3: From eqn. 20, solve $l_{i}(s)$ and $f_{i}(s)$ with free parameters, where $g_{i}(s), i=1, \ldots, n$ are determined by the desired closed-loop poles for each channel.

Step 4: By satisfying eqn. 22, determine the free mined.

Step 5: Obtain the controller $C(s)$ as in eqn. 21.

Remark: Moreover, we can minimise $\left\|1-s_{i}\right\|_{\infty}, i=1$, $\ldots, n$ to obtain the robustness optimisation of the system with desired pole-zero assignment, i.e., we can predict the maximum slope

$$
\rho_{i_{\max }}=\frac{1}{\min \left\|1-s_{i}\right\|_{x}}
$$

of the sector-bounded nonlinear uncertainties that can be tolerated in each channel of the multivariable feedback system. parameters of $l_{i}(s)$ in eqn. 19. And then $S(s)$ is also deter- 


$$
\left[\begin{array}{c}
\beta \\
\phi \\
\psi
\end{array}\right]=\left[\begin{array}{lllll}
1 & 0 & 0 & 0 & 0 \\
0 & 1 & 0 & 0 & 0 \\
0 & 0 & 0 & 0 & 1
\end{array}\right]\left[\begin{array}{c}
\beta \\
\phi \\
p \\
r \\
\psi
\end{array}\right]
$$

where $\beta$ is the sideslip angle (rad), $\phi$ is the rolling angle ( $\mathrm{rad}), p$ is the rolling $\mathrm{rate}(\mathrm{rad} / \mathrm{s}), r$ is the yaw $\mathrm{rate}(\mathrm{rad} / \mathrm{s}), \psi$ is the yaw angle (rad), $\delta_{f}$ is the direct lift control angle (rad), $\delta_{r}$ is the yaw rudder angle (rad), and $\delta_{v_{c}}$ is the direct sideforce control angle (rad).

The transfer matrix form of the system can be obtained as

$$
\left[\begin{array}{l}
\beta(s) \\
\phi(s) \\
\psi(s)
\end{array}\right]=\left[\begin{array}{lll}
p_{11}(s) & p_{12}(s) & p_{13}(s) \\
p_{21}(s) & p_{22}(s) & p_{23}(s) \\
p_{31}(s) & p_{32}(s) & p_{33}(s)
\end{array}\right]\left[\begin{array}{c}
\delta_{f}(s) \\
\delta_{r}(s) \\
\delta_{v_{c}}(s)
\end{array}\right]
$$

where

$$
\begin{aligned}
& p_{11}(s)=\frac{\beta(s)}{\delta_{f}(s)}=\frac{-0.09(s-1.2001)(s+44.6357)(s+0.63442)}{(s-2.1987)(s-0.10122)(s+2.0592)(s+3.9698)} \\
& p_{12}(s)=\frac{\beta(s)}{\delta_{r}(s)}=\frac{0.121(s-0.0080644)(s+131.941)(s+3.2726)}{(s-2.1987)(s-0.10122)(s+2.0592)(s+3.9698)} \\
& p_{13}(s)=\frac{\beta(s)}{\delta_{v_{c}}(s)}=\frac{0.081(s-76.0459)(s-0.0345)(s+2.9332)}{(s-2.1987)(s-0.10122)(s+2.0592)(s+3.9698)} \\
& p_{21}(s)=\frac{\phi(s)}{\delta_{f}(s)}=\frac{151(s-2.1035)(s+2.9206)}{(s-2.1987)(s-0.10122)(s+2.0592)(s+3.9698)} \\
& p_{22}(s)=\frac{\phi(s)}{\delta_{r}(s)}=\frac{46.2(s-5.5963)(s+5.4511)}{(s-2.1987)(s-0.10122)(s+2.0592)(s+3.9698)} \\
& p_{23}(s)=\frac{\phi(s)}{\delta_{v_{c}}(s)}=\frac{7.106(s+0.8846+j 6.9426)(s+0.8846-j 6.9426)}{(s-2.1987)(s-0.10122)(s+2.0592)(s+3.9698)} \\
& p_{31}(s)=\frac{\psi(s)}{\delta_{f}(s)}=\frac{3.654(s-1.6984)(s+1.5426+j 1.8985)(s+1.5426-j 1.8985)}{s(s-2.1987)(s-0.10122)(s+2.0592)(s+3.9698)} \\
& p_{32}(s)=\frac{\psi(s)}{\delta_{r}(s)}=\frac{-15.94(s+3.469)(s-0.0027+j 0.994)(s-0.0027-j 0.994)}{s(s-2.1987)(s-0.10122)(s+2.0592)(s+3.9698)} \\
& p_{33}(s)=\frac{\psi(s)}{\delta_{v_{c}}(s)}=\frac{6.206(s+3.1607)(s-0.0309+j 0.8309)(s-0.0309-j 0.8309)}{s(s-2.1987)(s-0.10122)(s+2.0592)(s+3.9698)}
\end{aligned}
$$

Let us consider the system illustrated in Fig. 1 with the nominal plant $P(s)$ described as above. We will synthesise a controller for:

(a) decoupling the nominal system;

(b) assigning five closed-loop system poles at $-1 / 2 \pm j(\sqrt{ }(3) / 2),-8,-12$ and -16 for channel 1 , at $-2 \pm j 2,-12$, -18 and -24 for channel 2 , at $-1 \pm j 1,-10,-15$ and -20 for channel 3 ;

(c) tracking the unit step reference signal for each channel;

(d) achieving the robust stabilisation of the sector-bounded nonlinear uncertainties

$$
\begin{aligned}
& \left|\Delta N_{1}[u(t), t]\right| \leqslant \frac{2}{3}\|u(t)\| \\
& \left|\Delta N_{2}[u(t), t]\right| \leqslant \frac{3}{5}\|u(t)\| \\
& \left|\Delta N_{3}[u(t), t]\right| \leqslant \frac{5}{7} \mid\|u(t)\| \forall\|u(t)\|<\infty
\end{aligned}
$$

Another design objective is to derive the maximum slopes $\rho_{1}, \rho_{2_{\max }}$ and $\rho_{3_{\max }}$ of the sector-bounded nonlinear uncertainties that can be tolerated in each channel of the feedback system under requirements $(a),(b)$ and $(c)$.

Solution: Firstly, we derive the conditions for achieving internal stability. Performing the factorisations of $P(s)$, we obtain $P(s)=A^{-1}(s) B(s)=B_{1}(s) A_{1}^{-1}(s)$, and

$$
A^{-1}(s)=\left[\begin{array}{lll}
a_{11}(s) & a_{12}(s) & a_{13}(s) \\
a_{21}(s) & a_{22}(s) & a_{23}(s) \\
a_{31}(s) & a_{32}(s) & a_{33}(s)
\end{array}\right] \quad B_{1}^{-1}(s)=\left[\begin{array}{lll}
b_{11}(s) & b_{12}(s) & b_{13}(s) \\
b_{21}(s) & b_{22}(s) & b_{23}(s) \\
b_{31}(s) & b_{32}(s) & b_{33}(s)
\end{array}\right]
$$

where

$$
a_{11}(s)=\frac{(s+4.1109)(s+2.9701)(s+0.8214)(s-0.0156)}{(s-0.1012)(s-2.1987)(s+3.9698)(s+2.0592) D(s)}
$$




$$
\begin{aligned}
& a_{12}(s)=\frac{0.3477(s+3.1143)(s+0.8631)(s+0.2136)}{(s-0.1012)(s-2.1987)(s+3.9698)(s+2.0592) D(s)} \\
& a_{13}(s)=\frac{0.0764(s-4.344)(s+2.946)(s+0.0971)}{(s-0.1012)(s-2.1987)(s+3.9698)(s+2.0592) D(s)} \\
& a_{21}(s)=\frac{-0.1188(s+556.51)(s+4.107)(s+0.9785)}{(s-0.1012)(s-2.1987)(s+3.9698)(s+2.0592) D(s)} \\
& a_{22}(s)=\frac{(s-2.6877)(s+1.4582)(s+4.4471+j 1.7138)(s+4.4471-j 1.7138)}{(s-0.1012)(s-2.1987)(s+3.9698)(s+2.0592) D(s)} \\
& a_{23}(s)=\frac{0.9168(s+5.8937)(s-1.4404)(s-0.2547)}{(s-0.1012)(s-2.1987)(s+3.9698)(s+2.0592) D(s)} \\
& a_{31}(s)=\frac{-0.1639(s+51.846)(s+4.0898)(s+2.4848)(s+0.02996)}{s(s-0.1012)(s-2.1987)(s+3.9698)(s+2.0592) D(s)} \\
& a_{32}(s)=\frac{0.7017(s-3.0011)(s+4.2938)(s+2.2135)(s+0.0842)}{s(s-0.1012)(s-2.1987)(s+3.9698)(s+2.0592) D(s)} \\
& a_{33}(s)=\frac{(s-2.1827)(s+3.7006)(s+3.2746)(s+22.2119)(s-0.1020)}{s(s-0.1012)(s-2.1987)(s+3.9698)(s+2.0592) D(s)} \\
& b_{11}(s)=\frac{400}{(s+1)(s+2)(s+3)(s+5)} \\
& b_{33}(s)=\frac{-22.429}{(s+3)(s+4)(s+5)} \\
& b_{12}(s)=\frac{-2.0421(s+3.8842)(s-19.167)}{D(s)} \\
& b_{33}(s)=\frac{-0.9925(s+3.8398)(s+312.66)}{D+1)(s+2)(s+3)(s+4)(s+5)} \\
& b_{13}(s)=\frac{-2.8824}{(s+3)(s+4)(s+5)} \\
& b_{21}(s)=\frac{-911}{(s+1)(s+2)(s+3)(s+5)} \\
& b_{22}(s)=\frac{-0.8545(s+3.8339)(s+130.91)}{D(s)} \\
& (s+3)(s+4)(s+5) \\
& (s+1)(s+2)(s+3)(s+5) \\
& (s)
\end{aligned}
$$

Then we obtain $\alpha_{i}(s)$ and $\beta_{i}(s)$ for $i=1,2,3$ as follows:

$$
\begin{aligned}
& x_{1}(s)=\alpha_{2}(s)=(s-2.1987)(s-0.10122) \\
& x_{3}(s)=s(s-2.1987)(s-0.10122) \\
& \beta_{1}(s)=\beta_{2}(s)=\beta_{3}(s)=1
\end{aligned}
$$

To satisfy the requirements $(a),(b)$ and $(c)$, the conditions (ii) and (iii) of Lemma 2 , and the requirements of proper controller and causality of the closed-loop system, since $\beta_{1}(s)=1, \beta_{2}(s)=1$, and $\beta_{3}(s)=1$, we choose $l_{1}(s)=s^{2}$ $+39.2999 s+\mu_{11}, l_{2}(s)=s^{2}+60.2999 s+\mu_{21}$, and $l_{3}(s)=s^{2}+49.2999 s+\mu_{31}$, with one undetermined parameter, respectively. Then the sensitivity matrix is chosen as

$$
S(s)=\left[\begin{array}{ccc}
s_{1}(s) & 0 & 0 \\
0 & s_{2}(s) & 0 \\
0 & 0 & s_{3}(s)
\end{array}\right]
$$

IEE PROCEEDINGS-D, Vol. 139, No. 3, MAY 1992 
where

$$
\begin{aligned}
& s_{1}(s)=\frac{s\left(s^{2}+39.2999 s+\mu_{11}\right)(s-0.10122)(s-2.1987)}{(s+8)(s+12)(s+16)\left(s^{2}+s+1\right)} \\
& s_{2}(s)=\frac{s\left(s^{2}+60.2999 s+\mu_{21}\right)(s-0.10122)(s-2.1987)}{(s+12)(s+18)(s+24)\left(s^{2}+4 s+8\right)} \\
& s_{3}(s)=\frac{s\left(s^{2}+49.2999 s+\mu_{31}\right)(s-0.10122)(s-2.1987)}{(s+10)(s+15)(s+20)\left(s^{2}+2 s+2\right)}
\end{aligned}
$$

For satisfying the stability robustness requirement, we have

$$
\begin{aligned}
& \frac{2}{3} \sup _{w \in[0, \infty]}\left|1-s_{1}(j \omega)\right|<1, \quad 41<\mu_{11}<244 \\
& \frac{3}{5} \sup _{\omega \in[0, \infty]}\left|1-s_{2}(j \omega)\right|<1, \quad-46<\mu_{21}<903 \\
& \frac{5}{7} \sup _{\omega \in[0, \infty]}\left|1-s_{3}(j \omega)\right|<1, \quad 164<\mu_{31}<344
\end{aligned}
$$

And the corresponding controller can be obtained as

$$
C(s)=P^{-1}(s) S^{-1}(s)(I-S(s))=\left[\begin{array}{lll}
c_{11}(s) & c_{12}(s) & c_{13}(s) \\
c_{21}(s) & c_{22}(s) & c_{23}(s) \\
c_{31}(s) & c_{32}(s) & c_{33}(s)
\end{array}\right]
$$

where

$$
\begin{aligned}
c_{11}(s)= & \frac{400(s-0.1299)}{s(s-0.10122)(s-2.1987)} \\
& \times \frac{\left(543.1641-\mu_{11}\right) s^{3}+\left(1979.2537+2.2999 \mu_{11}\right) s^{2}+\left(1952-0.2226 \mu_{11}\right) s+1536}{s^{2}+39.2999 s+\mu_{11}} \\
c_{12}(s)= & \frac{-2.0421(s+1.5353+j 2.2983)(s+1.5353-j 2.2983)}{s(s-0.10122)(s-2.1987)} \\
& \times \frac{\left(1298.462-\mu_{21}\right) s^{3}+\left(9346.58+2.2999 \mu_{21}\right) s^{2}+\left(28224-0.2226 \mu_{21}\right) s+41472}{s^{2}+60.2999 s+\mu_{21}} \\
c_{13}(s)= & \frac{-2.8824(s-139.7452)}{(s-0.10122)(s-2.1987)} \\
& \times \frac{\left(855.1633-\mu_{31}\right) s^{3}+\left(4379.0282+2.2999 \mu_{31}\right) s^{2}+\left(7300-0.2226 \mu_{31}\right) s+6300}{s^{2}+49.2999 s+\mu_{31}} \\
c_{21}(s)= & \frac{-911.14(s+0.2087)}{s(s-0.10122)(s-2.1987)} \quad s^{2}+49.2999 s+\mu_{31} \\
& \times \frac{\left(543.1641-\mu_{11}\right) s^{3}+\left(1979.2537+2.2999 \mu_{11}\right) s^{2}+\left(1952-0.2226 \mu_{11}\right) s+1536}{s^{2}+39.2999 s+\mu_{11}} \\
c_{22}(s)= & \frac{-0.8545(s+7.6692)(s-5.4223)}{s(s-0.10122)(s-2.1987)} \\
& \times \frac{\left(1298.462-\mu_{21}\right) s^{3}+\left(9346.58+2.2999 \mu_{21}\right) s^{2}+\left(28224-0.2226 \mu_{21}\right) s+41472}{s^{2}+60.2999 s+\mu_{21}} \\
c_{31}(s)= & \frac{-2575.8(s+0.3527)}{s(s-0.10122)(s-2.1987)} \quad \frac{\left(543.1641-\mu_{11}\right) s^{3}+\left(1979.2537+2.2999 \mu_{11}\right) s^{2}+\left(1952-0.2226 \mu_{11}\right) s+1536}{s^{2}+39.2999 s+\mu_{11}} \\
c_{23}(s)= & \frac{12.871(s-70.1873)}{(s-0.10122)(s-2.1987)} \quad \\
& \frac{\left(855.1633-\mu_{31}\right) s^{3}+\left(4379.0282+2.2999 \mu_{13}\right) s^{2}+\left(7300-0.2226 \mu_{31}\right) s+6300}{s^{2}} \\
&
\end{aligned}
$$




$$
\begin{aligned}
c_{32}(s)= & \frac{-0.9925(s+12.3354)(s-8.2054)}{s(s-0.10122)(s-2.1987)} \\
& \times \frac{\left(1298.462-\mu_{21}\right) s^{3}+\left(9346.58+2.2999 \mu_{21}\right) s^{2}+\left(28224-0.2226 \mu_{21}\right) s+41472}{s^{2}+60.2999 s+\mu_{21}} \\
c_{33}(s)= & \frac{-22.429(s+115.2201)}{(s-0.10122)(s-2.1987)} \\
& \times \frac{\left(855.1633-\mu_{31}\right) s^{3}+\left(4379.0282+2.2999 \mu_{31}\right) s^{2}+\left(7300-0.2226 \mu_{31}\right) s+6300}{s^{2}+49.2999 s+\mu_{31}}
\end{aligned}
$$

Moreover,

$$
\begin{array}{ll}
\min _{C(s)} \sup _{\omega \in[0, \infty]}\left|1-s_{1}(j \omega)\right|=1.3719, & \mu_{11}=153 \\
\min _{C(s)} \sup _{\omega \in[0, \infty]}\left|1-s_{2}(j \omega)\right|=1.4252, & \mu_{21}=385 \\
\min _{C(s)} \sup _{\omega \in[0, \infty]}\left|1-s_{3}(j \omega)\right|=1.3625, & \mu_{31}=254
\end{array}
$$

And then the maximum slopes of the sector-bounded nonlinear uncertainties that can be tolerated in this system are $\quad \rho_{1_{\max }}=(1 / 1.3719)=0.7289, \quad \rho_{2_{\max }}=$ $(1 / 1.4252)=0.7017$, and $\rho_{3_{\max }}=(1 / 1.3625)=0.7339$. That
is if no nonlinear uncertainty exists $-\Delta N[u(t), t]=0$, for all $t \in[0 . \infty]$ - and we choose the controller as in eqn. 32 with arbitrary real $\mu_{11}, \mu_{21}$ and $\mu_{31}$, then the feedback system in Fig. 1 achieves internal stability with desired pole-zero assignment. And if there exist nonlinear uncertainties within the sector bound with slopes $\rho_{1}=(2 / 3)$, $\rho_{2}=(3 / 5)$ and $\rho_{3}=(5 / 7)$, we choose the controller as in eqn. 32 , by satisfying inequalities eqns. 29,30 and 31 , then the feedback system in Fig. 1 also achieves robust stability. Moreover, when we choose the controller as in eqn. 32 with $\mu_{11}=153, \mu_{21}=385$ and $\mu_{31}=254$, then the feedback system in Fig. 1 can tolerate the nonlinear uncertainties within the maximum sector bound with slopes $\rho_{1_{\max }}=0.7289, \rho_{2_{\max }}=0.7017$ and $\rho_{3_{\max }}=0.7339$.

The Nyquist diagrams of the loop gain for channel 1 with $\mu_{11}=41,153$ and 244 are shown in Fig. 2 . Since the loop gain with $\mu_{11}=41$ and 244 , respectively, has two poles (2.1987 and 0.1012) in the right-half plane and from Fig. 2, the Nyquist diagram of the loop gain with $\mu_{11}=$ 41 and 244 , completely encircles twice in a counterclockwise direction, then the circle disk which intersects

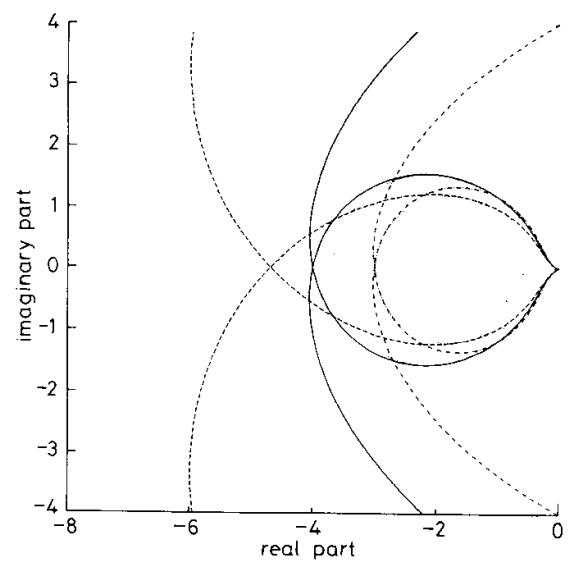

Fig. 2 Nyquist diagram of the loop gain for channel 1

$-\cdots \mu_{11}=41$ (dashed line)

$\mu_{\mathrm{t1}}=153$ (solid line)
$--\quad \quad \mu_{11}=244$ (dashdot line)

IEE PROCEEDINGS-D, Vol. 139, No. 3, MAY 1992

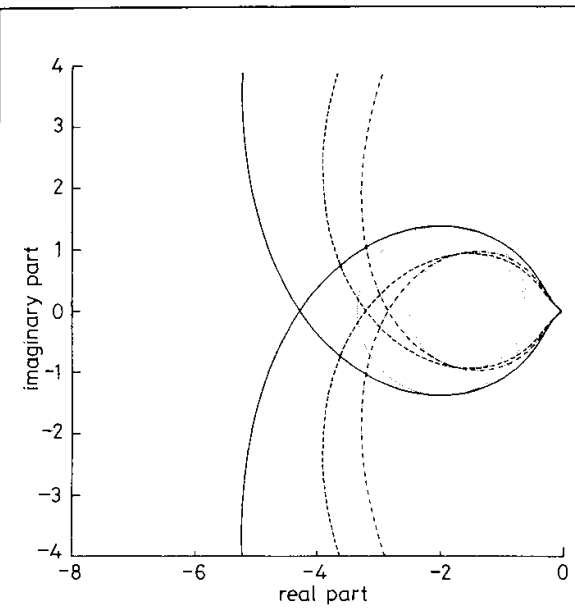

Fig. 3 Nyquist diagram of the loop gain for channel 2

$-\cdots \mu_{21}=-46$ (dashed line)

$-\quad \mu_{21}=385$ (solid line)

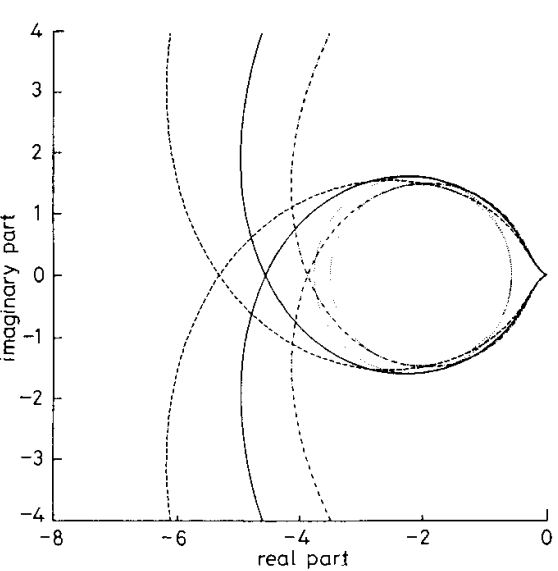

Fig. 4 Nyquist diagram of the loop gain for channel 3

$\ldots-\mu_{31}=164$ (dashed line)

$\begin{aligned} \mu_{31} & =254 \text { (solid line) } \\ \mu_{31} & =344 \text { (dashdot line) }\end{aligned}$ 
the real axis at boundary values $[-1 /(1-(2 / 3))]=-3$ and $[-1 /(1+(2 / 3))]=-0.6$ corresponds to the slope $\rho_{1}=(2 / 3)$ of the sector-bounded uncertainty $\Delta N_{1}[u(t), t]$ in the feedback system. Moreover, the loop gain for $\mu_{11}=153$ also has two poles (2.1987 and 0.1012$)$ in the right-half plane, and from Fig. 2 , the Nyquist diagram of the loop gain for $\mu_{11}=153$ completely encircles twice in counterclockwise direction, the circle disk which intersects the real axis at boundary values $[-1 /(1-0.7289)]=-3.6887$ and $[-1 /(1+0.7289)]=$ -0.5784 corresponding to the maximum slope $\rho_{1_{\max }}=$ 0.7289 of the sector-bounded uncertainty $\Delta N_{1}[u(t), t]$ that can be tolerated in the feedback system. These satisfy the circle criterion and Nyquist criterion, thus the robust stability is ensured. Similarly, the Nyquist diagrams of the loop gain for channels 2 and 3 with $\mu_{21}=-46,385$ and 903 and $\mu_{31}=164,254$ and 344 shown in Figs. 3 and 4 also satisfy the circle criterion and Nyquist criterion, thus the robust stability is ensured.

\section{Numerical algorithm for controller design}

The computer-aided-design package MATLAB has been used for the numerical computations in this paper. The numerical algorithm for controller design in the preceding Sections includes:

(a) conversion of a system from a state-space model to transfer function matrix form:

(b) left and right coprime factorisations of a transfer function matrix;

(c) inversion of a transfer function matrix;

(d) $H^{\infty}$-norm calculation of a transfer function.

For Item (a), an M-file [15] is written by using the state-space to transfer function conversion function of the MATLAB Control System Toolbox.

For Item $(b)$, the algorithm given in Reference 16 (Section 4.1) is adopted to design an M-file to obtain the left and right coprime factorisations of a transfer function matrix.

For Item (c), an M-file is written to construct the adjoint matrix and the determinant of a transfer function matrix by using the convolution function of the MATLAB toolbox. For a rational matrix which is proper and not strictly proper, a more efficient algorithm given in Reference 16 (Section 7.1) is used to design an M-file to obtain the inverse of a transfer function matrix.

For Item $(d)$, the algorithm given in Reference 17 is adopted to design an $\mathrm{M}$-file to calculate the $H^{\infty}$-norm of a transfer function by using the transfer function to statespace conversion function of the MATLAB control system toolbox and the eigenvalue function of the MATLAB toolbox.
A design criterion has been developed to simultaneously consider the performance and the stability robustness of a multivariable feedback system. Moreover, by minimising $H^{\infty}$-norm of each channel of the closed-loop transfer matrix, we can predict the maximum slope of the sector-bounded nonlinear uncertainties that can be tolerated in each channel of the feedback system. Since the requirements of internal stability are satisfied, this design algorithm performs appropriately, even if the plant is unstable and/or nonminimum phase.

\section{References}

1 CHEN, C.-T.: 'Linear system theory and design' (Holt, Rinehart \& Winston, 1984)

2 ASTROM, K.J., and WITTENMARK, B.: 'Computer controlled systems' (Prentice-Hall, 1990)

3 WOLOVICH, W.A.: 'Multipurpose controllers for multivariable systems', IEEE Trans., 1981, AC-26, pp. 162-170

4 SAEKS, R., and MURRAY, J.: 'Feedback system design: the tracking and disturbance rejection problem', IEEE Trans., 1981, AC-26, p. 203-217

5 CRUZ, J.B., FREUDENBERG, J.S., and LOOZE, D.P.: 'A relationship between sensitivity and stability of multivariable feedback systems', IEEE Trans., 1981, AC-26, pp. 66-74

6 DOYLE, J.C., and STEIN, G.: 'Multivariable feedback design: concepts for a classical/modern synthesis', IEEE Trans., 1981, AC-26, pp. $4-16$

7 CHEN, M.J., and DESOER, C.A.: 'The problem of guaranteeing robust disturbance rejection in linear multivariable feedback robust disturbance rejection in linear multion
systems', Int. J. Control, 1983., 37, pp. 305-313

systems', Int. J. Control, $1983 ., 37, \mathrm{pp} .305-313$
8 DICKMAN, A., and SIVAN, R.: 'On the robustness of multivariable feedback systems', IEEE Trans., 1985, AC-30, pp. 401-404

9 YEDAVALLI, R.K., and LIANG, Z.: 'Reduced conservatism in stability robustness bounds by state transformation', IEEE Trans. 1986, AC-31, pp. 863-866

10 ZHOU, $\mathrm{K}$, and KHARGONEKAR, P P. 'Stability robustness bounds for linear state space models with structured uncertainty', IEEE Trans., 1987, AC-32, pp. 621-623

11 HYLAND, D.C., and BERNSTEIN, D.S.: 'The majorant Lyapunov equation: a non-negative matrix equation for robust stability and performance of large scale systems', IEEE Trans., 1987, AC-32, pp. 1005-1013

12 COLLINS, E.G. Jr., and HYLAND, D.C.: 'Improved robust performance bounds in covariance majorant analysis'. Proc. 27th IEEE Conf. Decision Contr., 1988, pp. 2188-2193

13 SAFONOV, M.G., and CHEN, B.S.: 'Multivariable stability-margin optimisation with decoupling and output regulation', IEE Proc. D, optimisation with decoupling and output regulati
Control Theory \& Appl, 1982, 129, pp. 276-282

14 KANAI, K., and UCHIKADO, S.: 'An adaptive flight control system design for $\mathrm{CCV}$ with an unknown interactor matrix', $J$. $J \boldsymbol{n}$ Aeronaut. \& Astronaut. Assoc., 1986, 34, (387), pp. 211-221

15 'PC-MATLAB user's guide, version 3.5' (The Mathworks, Inc South Natick, MA, USA, 1990)

16 FRANCIS, B.A.: 'A course in $H^{\infty}$ control theory' (Springer-Verlag, 1987)

17 DOYLE, J.C., GLOVER, K., KHARGONEKAR, P.P., and FRANCIS, B.A.: 'State-space solutions to standard $H^{2}$ and $H^{\infty}$ control problems', IEEE Trans., 1989, AC-34, pp. 831-847 